\title{
Building of an immunosensor: How can the composition and structure of the thiol attachment layer affect the immunosensor efficiency?
}

\author{
Elisabeth Briand ${ }^{a}$, Michèle Salmain ${ }^{b}$, Jean-Marie Herry ${ }^{c}$, Hubert Perrot ${ }^{d,}$ \\ Chantal Compère ${ }^{\mathrm{e}}$ and Claire-Marie Pradier ${ }^{\mathrm{a},{ }^{*}}$
}

\begin{abstract}
aLaboratoire de Réactivité de Surface, UMR CNRS 7609, Université Pierre et Marie Curie, 4 Place Jussieu, 75252 Paris Cedex 05, France

bLaboratoire de Chimie et Biochimie des Complexes Moléculaires, UMR CNRS 7576, Ecole Nationale Supérieure de Chimie, 11 Rue Pierre et Marie Curie, 75231 Paris Cedex 05, France

'Unité de Bioadhésion et d'Hygiène des Matériaux INRA 91300 Massy France

'Laboratoire des Interfaces et Systèmes Electrochimiques, UPR 15, Case 133, 4 place Jussieu, 75252 Paris Cedex 05, France

eDépartement Essais et Recherches Technologiques, Interface et Capteurs, IFREMER, Centre de Brest, BP 70, 29280 Plouzané, France
\end{abstract}

*: Corresponding author : Tel.: +33 1442755 33; fax: +33 1442760 33. Email : pradier@ccr.jussieu.fr

\begin{abstract}
Immunosensors, based on the immobilization of a model rabbit antibody on mixed self-assembled monolayers and Protein A as a linking agent on gold transducers, were elaborated and characterized at each step by modulated polarization-infrared spectroscopy (PM-IRRAS) and occasionally by atomic force microscopy (AFM) and quartz crystal microbalance (QCM). By testing two different mixed SAMs comprising 11-mercaptoundecanoic acid (MUA), together with either decanethiol $(\mathrm{C} 9 \mathrm{CH} 3)$ or mercaptohexanol $(\mathrm{C} 6 \mathrm{OH})$, the role of the chemical composition and structure of the antibody attachment layer upon the sensor performance was demonstrated.
\end{abstract}

Keywords: Mixed SAM's; Immunosensor; Thiol attachment; IRRAS; QCM 


\section{Introduction}

Many research fields involve the study of interaction of biomolecules with solid surfaces in particular for new biosensor elaboration. A biosensor can be described as a sensitive interface including a bioreceptor coupled with a transducer able to detect binding events between the bioreceptor and the analyte. Biosensors provide a rapid and convenient alternative to conventional analytical methods for detecting, and in some cases measuring, an analyte in a complex medium. Different classes of biosensors are classically distinguished, among them immunosensors that exploit the ability of an antibody to recognize its associated antigen in a very complex medium ${ }^{1}$.

The quality of the biointerface governs both the sensitivity and the specificity of the biosensor. Therefore, its design is the key step during the biosensor elaboration process. Ideally, the bioreceptor should specifically recognize and bind target species at the lowest possible concentration. It is well known that proteins, and antigens in particular, may loose part of their biological activity when immobilized on a surface due to a combination of two factors: change in conformation and/or unfavourable orientation of the molecule ${ }^{2}$. As a consequence, controlling both the amount and orientation of the bioreceptor on the transducer while preserving its bioactivity is the most critical issue in biosensor research.

Immobilization of bioreceptors (in particular antibodies) via self-assembled monolayers (SAMs) or mixed SAMs enables to investigate biorecognition phenomena at a molecular level and fulfils the criteria mentioned above. Let us remind that alkylthiols spontaneously chemisorb on gold surfaces and form densely packed, crystalline-like thiolate films in a very reproducible manner ${ }^{3-7}$. When dealing with $\omega$-functionalized thiols, various chemical moieties can thus be strongly bound at the solid-liquid interface, and materials with new surface properties can be designed for the binding of biomolecules ${ }^{8-10}$. In some cases, the use of mixed SAMs, formed for instance by co-adsorption of mixtures of two thiols, has been shown to prevent denaturation and thus improve the bioactivity of a protein immobilized on such layers in comparison with the protein immobilized on a pure SAM ${ }^{11-14}$. Mixed SAMs are generally constituted of one thiolate with a functional headgroup (like a carboxylic acid) at a low mole fraction and of another "diluting” thiolate at a high mole fraction. The second thiol first reduces the surface concentration of functional groups and thus minimizes steric hindrance, partial denaturation of the protein ${ }^{13}$ and non specific interactions that can produce 
interference signals ${ }^{11,12}$. Second, the diluting thiolate can also be used to tailor the overall physico-chemical properties of the interface (such as its hydrophobic / hydrophilic character). Characterization of surfaces covered with mixed SAMs is still the subject of numerous studies $15-18$

In this study, we report the construction of an immunosensor, taking the rabbit IgG / antirabbit IgG couple as a model system. The biointerface consisted of a layer of antibody immobilized by bioaffinity onto Protein A, often used as a linker agent for biosensor elaboration $^{19-21}$; Protein A was covalently linked to two mixed SAM systems where 11mercaptoundecanoic acid is the "active" thiol. We have found that the nature of the diluting thiolate had a noticeable influence not only on the overall amount of immobilized Protein A and but also on its affinity towards rabbit IgG and finally on the properties on the resulting immunosensor.

\section{Materials and methods}

\subsection{Chemicals}

n-Decanethiol (C9CH3), 6-mercaptohexanol (C6OH), 11-mercaptoundecanoic acid (MUA), N-hydroxysuccinimide (NHS), 1-(3-dimethylaminopropyl)-N'-ethylcarbodiimide hydrochloride (EDC) were purchased from Aldrich (St-Quentin Fallavier, France). Rabbit IgG, goat anti-rabbit IgG, goat anti-mouse IgG and bovine serum albumin (BSA) were purchased from Pierce (Perbio, Brebières, France). All solvents were reagent-grade. Reagents were used without any further purification. Experiments were carried out at room temperature.

\subsection{Formation of the mixed SAMs}

Glass substrates $(11 \times 11 \mathrm{~mm})$ coated successively with $50 \mathrm{~nm}$ thick layer of chromium and a $200 \mathrm{~nm}$ thick layer of gold were purchased from Arrandee (Werther, Germany). The goldcoated substrates were annealed in a butane flame to ensure a good crystallinity of the topmost layers, as recommended by the company, and rinsed in a bath of absolute ethanol during 15 min before adsorption. The substrates were immersed in binary mixtures of $2.5 \mathrm{mM}$ of MUA and $7.5 \mathrm{mM}$ of $\mathrm{C} 9 \mathrm{CH} 3$ or $7.5 \mathrm{mM}$ of $\mathrm{C} 6 \mathrm{OH}$ in absolute ethanol for $3 \mathrm{~h}$ and thorough rinsed in ethanol and dried under a flow of clean air. 


\subsection{Covalent immobilization of Protein A}

The substrates were treated with a solution of NHS (20 mM) and EDC (10 mM) in ultrapure water for $2 \mathrm{~h}$ following by an immersion in a solution of Protein A (50 mg/L) in $10 \mathrm{mM}$ PBS pH 7.4 for 2 h. The residual NHS esters were blocked with $1 \mathrm{M}$ ethanolamine $\mathrm{pH} 9.0$ for 20 min. After washing with ultrapure water, the substrates were finally immersed in a $1 \%(\mathrm{w} / \mathrm{v})$ solution of BSA in $10 \mathrm{mM}$ PBS pH 7.4 for $2 \mathrm{~h}$.

\subsection{Binding of antibody}

Protein A-coated substrates were immersed in a solution of rabbit IgG (100 mg/L) in PBS for $1 \mathrm{~h}$, washed with water and dried under a flow of clean air

\subsection{Tests of specificity}

Goat anti-mouse IgG or goat IgG (non specific antigen) $(10 \mathrm{mg} / \mathrm{L}$ or $30 \mathrm{mg} / \mathrm{L}$ in PBS respectively; $150 \mu \mathrm{L}$ ) was spotted on the immunosensor and incubated for $1 \mathrm{~h}$. After extensive washing with water and PM-IRRAS analysis, goat anti-rabbit IgG (specific antigen) (10 or $30 \mathrm{mg} / \mathrm{L}$ in PBS; $150 \mu \mathrm{L}$ ) was spotted on the immunosensor and incubated for $1 \mathrm{~h}$.

\subsection{PM-IRRAS measurements}

The FTIR instrument used in our experiment is a commercial NICOLET Nexus spectrometer. The external beam was focused on the sample with a mirror, at an optimal incident angle of $75^{\circ}$. A ZnSe grid polarizer and a ZnSe photoelastic modulator, modulating the incident beam between $\mathrm{p}$ and s polarizations (HINDS Instruments, PEM 90, modulation frequency $=37$ $\mathrm{kHz}$ ), were placed prior to the sample. The light reflected at the sample was then focussed on a nitrogen-cooled MCT detector. The sum and difference interferograms were processed and Fourier-transformed to yield the differential reflectivity $\Delta \mathrm{R} / \mathrm{R}=(\mathrm{Rp}-\mathrm{Rs}) /(\mathrm{Rp}+\mathrm{Rs})$ which is the PM-IRRAS signal. 64 scans were recorded at $8 \mathrm{~cm}^{-1}$ resolution for each spectrum.

\subsection{AFM imaging}

The AFM was a PicoLE by Molecular Imaging (Scientec, Palaiseau, France) used in an acoustic mode in air at $22^{\circ} \mathrm{C}$. Images of $500 \times 500 \mathrm{~nm}$ were obtained at $0.75 \mathrm{~Hz}$ with a resolution $512 * 512$ pixels, with the following characteristics: 
Probe Characteristics Ultrasharp NSC15/noAI. Radius of curvature less than $10 \mathrm{~nm}$. Tip height: $15 \ldots 20 \mu \mathrm{m}$. Full tip cone angle less than $30^{\circ}$. Cantilever length: $\mathrm{L} \pm 5 \mu \mathrm{m}$ : 230 .

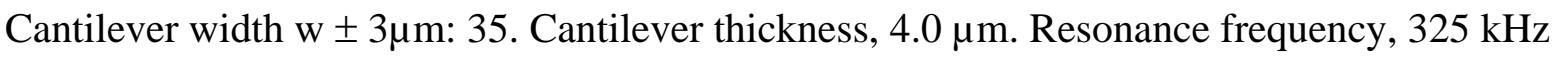
Force constant $40 \mathrm{~N} / \mathrm{m}$.

\subsection{QCM measurements}

AT-cut planar quartz crystals (14 mm diameter) with a $9 \mathrm{MHz}$ nominal resonance frequency (Matel Fondhal France) were used. Two identical gold electrodes, $2000 \AA$ thick and $5 \mathrm{~mm}$ in diameter, were deposited by evaporation techniques on both sides of crystals with a chromium underlayer. The resonators were connected with a silver conducting paste, through wires, to a BNC adaptator. A home-made oscillator was designed to drive the crystal at $27 \mathrm{MHz}$ which corresponds to the third overtone of the quartz resonator. To improve the stability, all the electronic oscillator components were temperature-controlled by a Watlow heater current monitor with a stability better than $0.1 \mathrm{~K}$. An experimental cell was developed: the crystal was mounted between two O-ring seals inserted in a Perspex cell. Only one face of the quartz was in contact with the solutions. The cell volume was $50 \mu \mathrm{L}$. The apparatus included a P1 micropump (Pharmacia) to ensure a $60 \mu \mathrm{L} / \mathrm{min}$ constant flowrate of the solutions. The experiments were performed at $25^{\circ} \mathrm{C}$, the room temperature. The experimental QCM setup consisted of the $27 \mathrm{MHz}$ QCM and a frequency counter PM 6685.

\section{Results and Discussion}

The successive steps of biointerface assembling are depicted in Fig. 1. Step 1 is the coadsorption of two thiols, MUA and $\mathrm{C9CH3}$ or $\mathrm{MUA}$ and $\mathrm{C6OH}$, at a constant 1 to 3 proportion, to form two different mixed SAMs; step 2 is the binding of Protein A by covalent linkage to the carboxylic acid function of MUA thiolate after activation by NHS and EDC; step 3 is the blocking of protein non-specific binding sites by BSA; step 4 is the immobilization of rabbit IgG antibody by bioaffinity. The two mixed SAM systems were systematically compared at each step.

The gold surfaces were characterized by PM-IRRAS providing chemical and structural information about thin organic films deposited on gold surfaces. Moreover AFM was used for gaining additional structural information on the mixed SAMs and the Protein A layers. QCM, 
performed in the flow-through mode, gave useful quantitative data on the amount of bound proteins.

Results will be presented and discussed step by step.

\subsection{Surface characterization of mixed SAMs}

Fig. 2a displays the PM-IRRAS spectrum of the gold surface after immersion in a mixture of MUA and C9CH3, rinsing in ethanol and drying under $\mathrm{N}_{2}$ flow. Four typical bands appeared in the $v(\mathrm{C}-\mathrm{H})$ region, at 2855, 2923 and 2877, $2962 \mathrm{~cm}^{-1}$, corresponding to the symmetric and asymmetric stretching vibrations of the chain $\mathrm{CH}_{2}$ and terminal $\mathrm{CH}_{3}$ respectively. The position of the $\mathrm{CH}_{2}$ asymmetric stretching vibration band at $2923 \mathrm{~cm}^{-1}$ is typical of a densely packed, quasi-crystalline arrangement of the alkyl chains thiolates ${ }^{22}$. Vibration bands in the lower wavenumber range were also observed at 1400 and $1700 \mathrm{~cm}^{-1}$, showing the presence of acidic groups, mainly in the deprotonated form. These findings provide good evidence that adsorption of the mixed SAM of MUA and decanethiol did occur.

Fig. 2b displays the same IR region of the surface spectrum after immersion in the mixture of MUA and $\mathrm{C} 6 \mathrm{OH}$, rinsing in ethanol and drying under $\mathrm{N}_{2}$ flow. This time, as expected, only the bands characteristic of the $\mathrm{CH}_{2}$ groups were observed 2853 and $2929 \mathrm{~cm}^{-1}$. The wavenumber of the symmetric and asymmetric $v(\mathrm{C}-\mathrm{H})$, slightly higher than for the MUA/C9CH3 mixed SAM, indicates a more disordered layer. This may be related to the difference of alkyl chain lengths between MUA and $\mathrm{C6OH}$ that does not favour self-assembly of these thiolates and induces disorder within the thin film. Note that the pure $\mathrm{C} 9 \mathrm{CH} 3$ and $\mathrm{C} 6 \mathrm{OH}$ SAM layers were also prepared and analysed, and the position of the two $v(\mathrm{C}-\mathrm{H})$ bands of the $\mathrm{CH}_{2}$ groups was 2853/2924 and 2857/2931 $\mathrm{cm}^{-1}$ respectively (spectra not shown).

Here again, bands at ca 1400, 1600 and $1700 \mathrm{~cm}^{-1}$, attributed to the symmetric, asymmetric $\mathrm{COO}^{-}$and $\mathrm{C}=\mathrm{O}$ stretch vibrations, prove the presence of adsorbed MUA for which the carboxyl group is both in the protonated and deprotonated forms. Although the comparison of the intensity of the carboxylic acid vibration bands for the two mixed SAMs is difficult as the IR measurements were done at two different periods of time, co-adsorption of MUA with $\mathrm{C6OH}$ always led to a slightly higher amount of acidic thiolates than co-adsorption with C9CH3. Note also that, for similar surface concentrations in MUA, changes in the orientation 
of the $\mathrm{C}=\mathrm{O}$ and $\mathrm{COO}^{-}$groups, for instance due to interactions with adjacent groups, may also account for variations in the stretching vibration intensities ${ }^{23}$.

The thiol-modified surfaces were then submitted to AFM. We first checked the presence of large planar areas on clean gold surfaces (data not shown) that allowed us to study the surface functionalization by formation of SAMs followed by the binding of protein A. The scan size for the images is then $500 \times 500 \mathrm{~nm}$. Figure 3a \& b show the topographic and phase images (figure inset) of the gold surfaces after co-adsorption of mixtures of MUA and C9CH3 or MUA and C6OH, respectively. While Fig. 3a suggests that the MUA/C9CH3 SAM was rather homogeneous both from structural and chemical aspects, Fig. 3b indicated the existence of defects dispersed all over the surface for the mixed MUA/C6OH SAM. Some topographic cross-sections were made on the two surfaces. They clearly showed a difference in homogeneity between the two mixed SAMs. On the SAM containing $\mathrm{C} 6 \mathrm{OH}$, the white spots were about $1 \mathrm{~nm}$ high, with some higher defaults. On the SAM containing C9CH3, the various phase domains are separated by about $5 \AA$. Thus, no phase segregation apparently occurred when MUA was adsorbed together with the long chain thiol decanethiol, probably because of strong hydrophobic and/or Van der Waals interactions expected to favour good orientation and ordering of the chains on the surface ${ }^{24}$, whereas it is tempting to interpret the second set of images as a regular distribution of MUA domains (white spots) dispersed within the $\mathrm{C} 6 \mathrm{OH}$ layer. The spot size is 20 to $40 \mathrm{~nm}$ suggests some phase segregation between MUA and $\mathrm{C6OH}$. Previously reported AFM data on mixed SAMs, showed more heterogeneous layers, with phase segregation, when the chain lengths of adsorbed thiols differ by more than 3-4 carbons ${ }^{16,25}$.

Chemical and structural differences for these two mixed layers are expected to induce changes in their reactivities and this will be studied in the following.

\subsection{Adsorption of Protein A on the mixed SAMs}

The SAM-coated gold transducers were first treated with a mixture of NHS and EDC in water to yield intermediately surface $\mathrm{N}$-hydroxysuccinimide ester functions. The transducers were then immediately exposed to a buffered solution of Protein A under two different conditions, $50 \mathrm{mg} / \mathrm{L}, 2 \mathrm{~h}$ and $0.5 \mathrm{mg} / \mathrm{L}, 15 \mathrm{~min}$ respectively. The first set of conditions ensured the saturation of the surface by Protein A, before the further antibody binding step; the second 
value was chosen so as to yield a submonolayer of protein and get exploitable AFM images. After copious washing, the gold surfaces were analysed by PM-IRRAS. Protein A is expected to be immobilized in a covalent manner on the activated MUA acid terminal groups. Fig. 4 shows the PM-IRRAS spectra of the unsaturated PrA layers on the MUA/C9CH3 and MUA/C6OH SAM layers. Binding of Protein A was assessed by the presence of intense bands at 1550 and $1660 \mathrm{~cm}^{-1}$ assigned to the Amide II and I bands, respectively. Whatever the Protein A adsorption conditions, almost twice as much Protein A was adsorbed on the MUA/C9CH3 SAM compared to the other one as evaluated from the integrated area A of the peptide bands. Moreover, a sharp band was observed at $1743 \mathrm{~cm}^{-1}$ and was readily attributed to the $\mathrm{C}=\mathrm{O}$ stretch of remaining $\mathrm{N}$-hydroxysuccinimide ester functions. Treatment in ethanolamine solutions induced a disappearance of the residual ester bands and an additional increase of the amide ones (spectra not shown).

Topographic and phase AFM images of the Protein A layers are shown in Fig. 5A \& B; they both show that the nature of the imaged object has changed compared to the images of Fig. 4 . One observes marked differences between Fig. 5 A and B: the layer of Protein A on the mixed MUA/C9CH3 SAM was apparently more homogeneous and dense than on the other SAM. Very small defects, $10 \mathrm{~nm}$ wide, $1 \mathrm{~nm}$ high, - a topographic cross section is shown in inset, appeared within the layer and there were no large aggregates as if the protein was regularly dispersed all over the SAM. Conversely, the second set of images revealed strong heterogeneities, aggregates of 30 to almost $100 \mathrm{~nm}$ wide in addition to rather large "empty" areas when Protein A was bound to the MUA/C6OH layer. A topographic cross-section shows a detail of some of these spots. A simple calculation, taking into account the respective sizes of the cantilever and Protein A, leads to an apparent size for the protein equal to ca. 30 $\mathrm{nm}$. This suggests that, on the mixed MUA/C6OH system, Protein A was immobilized in single items or small clusters. The height of the white spots is less than $1 \mathrm{~nm}$, i.e. below the size of Protein A (ca. $3 \mathrm{~nm}^{26}$ ). Nevertheless, the images have been obtained in air-acoustic mode, and the force applied to the molecules may induce a deformation of the protein. The smaller coverage in Protein A on the mixed MUA/C6OH system (from PM-IRRAS data) and the more heterogeneous character of the layer might be explained by (1) disorder in the SAM layer and poor dispersion of the adsorbed MUA thiolates, yielding a low number of useful anchoring points for Protein A, and/or (2) additional physisorption of Protein A on the mixed SAM of MUA and C9CH3 by hydrophobic interaction the methyl-terminated thiolate. This 
assumption is reinforced by the common knowledge that hydrophilic OH-terminated SAMs are less prone to protein physisorption than methyl-terminated SAMs ${ }^{27-29}$.

\subsection{Binding of BSA}

This step aims at blocking fractions of the surface that are not covered with Protein A and where the antibody might bind in an uncontrolled way. Knowing that the antibody will not bind to a BSA layer and that BSA does not associate with Protein $\mathrm{A}^{26}$, this step is expected to ensure antibody immobilization strictly to Protein A. The transducers were treated with a solution of BSA and analysed by PM-IRRAS.

Depending on the nature of the underlying SAM layer, different results were obtained (see Fig. 6A \& B, spectra b). For the mixed MUA/C9CH3 underlayer, no increase of the amide bands was observed. As we have just seen that a large amount of Protein A was bound to the surface in this case, a straightforward explanation may be that there was no space left accessible for BSA to bind, although exchange of Protein A by BSA at the liquid/solid interface cannot be completely ruled out, as proteins cannot be distinguished from one another by IR spectroscopy. Conversely, for the mixed MUA/C6OH underlayer, a significant increase of the amide band integrated area was observed $(\mathrm{A}=10.8 \Delta \mathrm{A}=2.5$, calculated from the peak area after ethanolamine treatment) indicating that an appreciable amount of BSA, similar to that of Protein A, could still bind to the surface. Since, on this surface, the total amount of bound Protein A was much lower, there should be available space for binding of BSA.

However it still raises the question why Protein A yielded a full monolayer on the mixed SAM of MUA and C9CH3 and not on the mixed SAM of MUA and $\mathrm{C6OH}$. A reasonable assumption would be to consider two competitive binding mechanisms of Protein A on the surface. The first mechanism implies chemisorption through covalent linkage with the carboxyl end groups of MUA thiolate. The second mechanism implies non-specific adsorption by other types of interaction. This second mechanism may occur only for the mixed MUA/C9CH3 SAM because of favourable interactions with the terminal $\mathrm{CH}_{3}$ groups of the diluting thiolate. Conversely, the use of $\mathrm{C} 6 \mathrm{OH}$ as diluting thiolate may somehow protect the surface against non-specific physisorption of Protein A. It appears however that it does not protect the surface against physisorption of BSA again in apparent contradiction with the general low affinity of proteins for hydroxyl-terminated SAMs ${ }^{28,29}$. Silin et al. also 
established that BSA poorly binds to an OH-terminated SAM (16-mercaptohexadecanol) ${ }^{30}$. However, their experiments were carried out with long-chain SAMs that are known to be well-ordered with their OH-terminal groups at the liquid / solid interface. When it comes to shorter thiols (number of carbons < 10), SAMs are generally less well ordered, indeed supported by our own IR surface data. Therefore, this disorder may enable interactions between BSA molecules and the methylene groups of the $\mathrm{C} 6 \mathrm{OH}$ chains. Note eventually that other authors showed that some proteins having polar side-end groups may interact with $\mathrm{OH}$ terminated SAMs ${ }^{29}$; the difference of behaviour between Protein A and BSA in that respect should be related to their surface chemical composition, keeping in mind that multiple modes of interaction are generally involved as regards protein binding to solid surfaces ${ }^{31}$. Another point worth being considered to explain the lower amount of bound Protein $\mathrm{A}$ on the MUA/C6OH SAM is the aggregation of MUA chains on that surface that induces steric constraints and reduces accessibility to the MUA terminal functions.

To summarize, it is now clearly established that either Protein A binds to the mixed MUA/C9CH3 and MUA/C6OH SAMs by different mechanisms or that a fraction, ca. 50\%, of the MUA terminal functions are not accessible when aggregated. The question is now whether this has consequences upon antibody binding and the properties of the resulting immunosensor.

\subsection{Binding of rabbit IgG binding to PrA}

The formation of the antibody layer was accomplished by treatment of the Protein A-coated transducers with a solution of rabbit IgG in PBS (100 mg/L, $1 \mathrm{~h})$. PM-IRRAS spectra of the gold surfaces are shown in Fig. 6A \& B, spectra c, for the mixed SAM of MUA/C9CH3 and MUA/C6OH, respectively. The surface IR spectra recorded after exposure to Protein A and BSA are superimposed for comparison. A very large increase of the amide band integrated areas was observed in both cases, i.e. $\Delta \mathrm{A}=22.2$ and 19.5 a.u., for the mixed MUA/C9CH3 and MUA/C6OH SAMs, respectively, indicating that the amount of antibody was a little smaller on the latter one. When comparing with the amide bands area measured after binding of Protein A on both surfaces, the increase was about two-fold for the MUA/C9CH3 SAM and three-fold for the MUA/C6OH SAM. Considering the relative sizes of Protein A (MW = $36 \mathrm{kDa}$ ) and rabbit IgG (MW = $150 \mathrm{kDa})$, the binding capacity of Protein A immobilized on the mixed SAM of $\mathrm{MUA} / \mathrm{C} 6 \mathrm{OH}$ was of ca. 0.75 rabbit IgG per Protein $\mathrm{A}$, which is 
reasonable. The binding capacity of Protein A immobilized on the mixed MUA/C9CH3 SAM was only around 0.5 rabbit IgG per Protein A. These features seem to indicate that binding of rabbit IgG to Protein A is more efficient on the MUA/C6OH underlayer, and two possible reasons can be suggested:

- Either the fraction of physically bound Protein A, present only on the MUA/C9CH3 layer, is not as efficient for IgG binding as the chemically bound Protein A, or

- There is a true steric hindrance on the layer that limits the binding capacities of the immobilized Protein A when it forms a dense layer on the MUA/C9CH3 SAM.

QCM in situ flow-through measurements, presented in Fig. 7A \& B, confirmed the rapid binding of rabbit IgG to the layer of Protein A and showed this time that a slightly higher amount of rabbit IgG was bound for the mixed MUA/C6OH SAM ( $\Delta \mathrm{F}=484 \mathrm{~Hz}$ vs $440 \mathrm{~Hz}$ for the mixed MUA/C9CH3 SAM). This slight discrepancy between IR and QCM results may be due to the very different procedures used for the two experiments, IRRAS analysis being performed ex situ and QCM analysis being performed in situ using a flow-through set up, i.e. obviously different mass transport conditions.

Having shown that the amount of bound rabbit IgG was not directly related to the amount of Protein A, one may wonder whether working with an even more dilute layer of anchoring thiol and/or binding protein would still enable the binding of a similar amount of antibody. We then repeated this series of surface functionalisation steps starting from a mixture of MUA and $\mathrm{C6OH}$, with a mole fraction of MUA $\chi$ equal to 0.1 and compared the results with those obtained for the MUA/C6OH SAM $(\chi=0.25)$. From our previous work, we assume that the surface mole fraction of MUA is close to that in solution ${ }^{23}$. Binding of Protein A, BSA, and eventually antibody, on these two SAMs were estimated from QCM flow-through measurements. Frequency changes are summarized in Table 1.

One sees first that, when the MUA mole fraction was decreased from 0.25 to 0.1 , the amount of Protein A bound to the surface was reduced by one third. Second, a higher amount of BSA adsorbed for the mixed SAM with $\chi=0.1$, confirming that the OH-terminated SAM did prevent adsorption of Protein A but not of BSA; eventually, in spite of these changes in the protein layers, the amount of bound antibody was very similar on both surfaces (and probably corresponded to a full monolayer of antibody); this suggests that, on the mixed SAM with $\chi=$ 
0.25, only a fraction of the Protein A binding sites was occupied by IgG. Here again, the layer containing the lowest amount of Protein A was the most efficient in terms of IgG binding capacity (1.5 rabbit IgG per protein A for the mixed SAM $\chi=0.1$ versus 1 rabbit IgG per protein A for the other one). Very similarly, Jenkins et al. demonstrated the importance of steric factors in the immobilization of large proteins like antibodies; they built immunosensors by immobilizing biotinylated antibodies on mixed SAMs, and came to the conclusion that the sensitivity of the optimum sensor surface may not correspond to the highest density of immobilized antibody ${ }^{32}$.

\begin{tabular}{|l|l|l|l|}
\hline Type of SAM & + PrA & + BSA & + rabbit IgG \\
\hline MUA/C6OH, $\chi=0.1$ & 100 & 162 & 445 \\
\hline MUA/C6OH, $\chi=0.25$ & 150 & 36 & 442 \\
\hline
\end{tabular}

Table 1: QCM frequency changes $(\mathrm{Hz})$ after successive binding steps

The following steps were only investigated for the SAM of MUA/C6OH, $\chi=0.25$.

\subsection{Antigen recognition}

The final key step consisted in testing the recognition of non-specific or specific antigens on the two model immunosensors built from the mixed SAM of MUA/C9CH3 and MUA/C6OH. PM-IRRAS was again used to probe their respective behaviour (Fig. 8A \& B). After exposure to the non-specific antigen (anti-mouse IgG $30 \mathrm{mg} / \mathrm{L}, \mathrm{t}=1 \mathrm{~h}$ ), a decrease of the amide bands intensity was noticed for both immunosensors, probably owing to the desorption of loosely bound antibody. This desorption was larger for the mixed SAM of MUA and C9CH3, suggesting that some antibody molecules were only weakly bound to Protein A, maybe because the latter were partially denatured or only physisorbed to the SAM layer. Subsequent treatment with specific antigen (anti-rabbit IgG $20 \mathrm{mg} / \mathrm{L}, \mathrm{t}=1 \mathrm{~h}$ ) led to a large increase of the amide bands intensity, as a result of its specific recognition by the immobilized antibody.

Interesting is to consider the antibody binding capacity for the antigen calculated from the amide band integrated area increase (no correction for sizes difference is necessary as antigen and antibody have the same MW). The antibody binding capacity was equal to 1.4 antigen per antibody for the mixed SAM of MUA and $\mathrm{C} 6 \mathrm{OH}$ and 1.1 for the other mixed SAM. 


\section{Conclusion and outlook}

This systematic comparison of the biosensor surfaces all through the successive steps for antibody grafting and eventually antigen recognition leads to the following conclusions:

- Using a diluent thiol in combination with an "active" thiol of similar chain lengths leads to a well ordered mixed SAM with no significant phase segregation. Conversely, the mixed SAM composed on thiols of very different lengths is rather disordered with noticeable phase segregation.

- This difference of film structure has a dramatic consequence on the amount of immobilized Protein A and on the structure of the protein layer, as a saturated Protein A layer is reached on the mixed SAM of MUA and C9CH3 but not on the mixed SAM of MUA and $\mathrm{C} 6 \mathrm{OH}$ where Protein A molecules form a less dense layer. This in turn has a marked influence on the binding capacity of Protein A for the antibody but has no influence on the overall amount of immobilized antibody. Eventually, the binding capacity of the antibody for the antigen is better for the mixed SAM of MUA and $\mathrm{C6OH}$ even if the overall amount of antibody is still the same.

We may thus expect that real-world immunosensors built according to these two strategies would have different properties in terms of sensitivity and dynamic range.

\section{Acknowledgement}

The CNRS and IFREMER are thanked for financing EB PhD grant.

\section{References}

(1) Wink, T.; van Zuilen, S. J.; Bult, A.; van Bennkom, W. P. Analyst 1997, 122, $43 \mathrm{R}$.

(2) Norde, W. Adv. in Colloid and Interface Sci. 1986, 25, 267.

(3) Bain, C. D.; Troughton, E. B.; Tao, Y.-T.; Evall, J.; Whitesides, G. M.; Nuzzo, R. G.

J. Am. Chem. Soc. 1989, 111, 321.

(4) Nuzzo, R. G.; Dubois, L. H.; Allara, D. L. J. Am. Chem. Soc. 1990, 112, 558.

(5) Porter, A. J.; Bright, T. B.; Allara, D. L.; Chidsey, C. E. D. J. Am. Chem. Soc. 1987, 109, 3559.

(6) Truong, K. D.; Rowntree, P. A. J. Phys. Chem. 1996, 100, 19917.

(7) Whitesides, G. M.; Laibinis, P. E. Langmuir 1990, 6, 87.

(8) Lahiri, J.; Isaacs, L.; Whitesides, G. M. Anal. Chem. 1999, 71, 777. 
(9) Su, X.-L.; Li, Y. Biosensors and Bioelectronics 2004, 19, 563.

(10) Ulman, A. Chem. Rev. 1996, 96, 1533.

(11) Frederix, F.; Bonroy, K.; Laureyn, W.; Reekmans, G.; Campitelli, A.; Dehean, W.; Maes, G. Langmuir 2003, 19, 4351.

(12) Ge, B.; Lisdat, F. Anal. Chim. Acta 2002, 454, 53.

(13) Guiomar, A. J.; Guthrie, J. T.; Evans, S. D. Langmuir 1999, 15, 1198.

(14) Wirde, M.; Gelius, U.; Nyholm, L. Langmuir 1999, 15, 6370.

(15) Chen, S.; Li, L.; Boozer, C. L.; Jiang, S. Langmuir 2000, 16, 9287.

(16) Chen, S.; Li, L.; Boozer, C. L.; Jiang, S. J. Phys. Chem. B 2001, 105, 2975.

(17) Hobara D., S. T., Imabayashi S.-i., Kakiuchi T. Langmuir 1999, 15, 5073.

(18) Li L., C. S., Jiang S. Langmuir 2003, 19, 666.

(19) Babacan S., P. P., Letcher S., Rand A.G. Biosensors and Bioelectronics 2000, 15, 615.

(20) Oh B.-K., K. Y.-K., Lee W., Bae Y. M., Lee W. H., Choi J.-W. Biosensors and Bioelectronics 2003, 18, 605.

(21) Pribyl, J.; Hepel, M.; Halamek, J.; Skladal, P. Sensors and Actuators B: Chemical 2003, 91, 333.

(22) Nuzzo, R. G.; Dubois, L. H.; Allara, D. L. J. Am. Chem. Soc. 1990, 112, 558.

(23) Briand, E.; Salmain, M.; Compère, C.; Pradier, C. M. to be published, 2006.

(24) Clegg, R. S.; Hutchison, J. E. J. Am. Chem. Soc., 1999, 121, 5319.

(25) Kakiuchi, T.; Iida, M.; Gon, N.; Hobara, D.; Imabayashi, S. I.; Niki, K. Langmuir 2001, 17, 1599.

(26) Marxer, C. G.; Coen, M. C.; Schlapbach, L. J. Colloid and Interf. Sci. 2003, 261, 291.

(27) Malmsten, M. J. Colloid Inter.e Sci. 1994, 166, 333.

(28) Martins, M. C. L.; Ratner, B. D.; Barbosa, M. A. J. Biomed.Mater. Res. 2003, 67, 158.

(29) Sigal, G. B.; Mrksich, M.; Whitesides, G. M., J. Am. Chem. Soc., 1998, 120, 3464.

(30) Silin, V.; Weetall, H.; Vanderah, D. J. J. Colloid and Interf. Sci.,. 1997, 185, 94.

(31) Tengvall, P.; Lundström, I.; Lieberg, B. Biomaterials 1998, 19, 407.

(32) Vareiro, M. L. M.; Liu, J.; Knoll, W.; Zak, K.; Williams, D.; Jenkins, A. T. A. Anal. Chem. 2005, 77, 2426. 
Figure 1: Schematic representation of the successive steps towards the immobilization of antibody

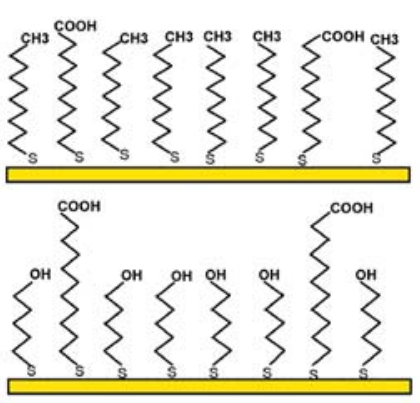

Step 1

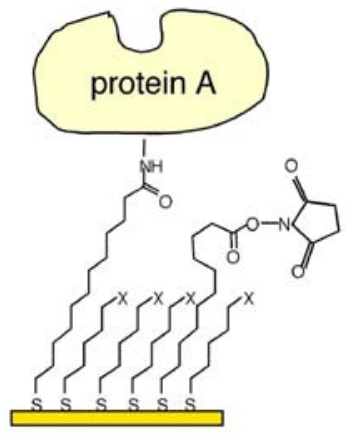

Step 2

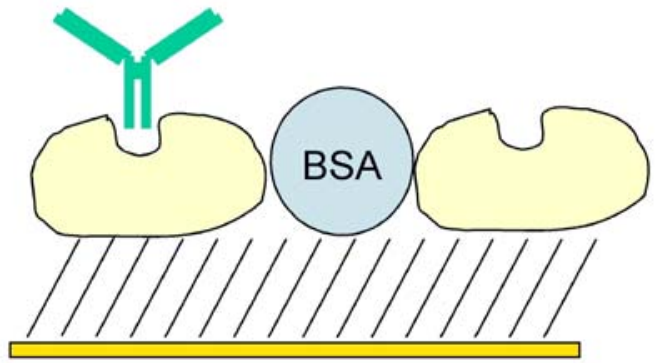

Steps $3 \& 4$

Figure 2: FT-IRRAS spectra of the gold surfaces after immersion in mixtures of (a) MUA and C9CH3; (b) MUA and C6OH. Samples were rinsed in ethanol and dried before analysis.
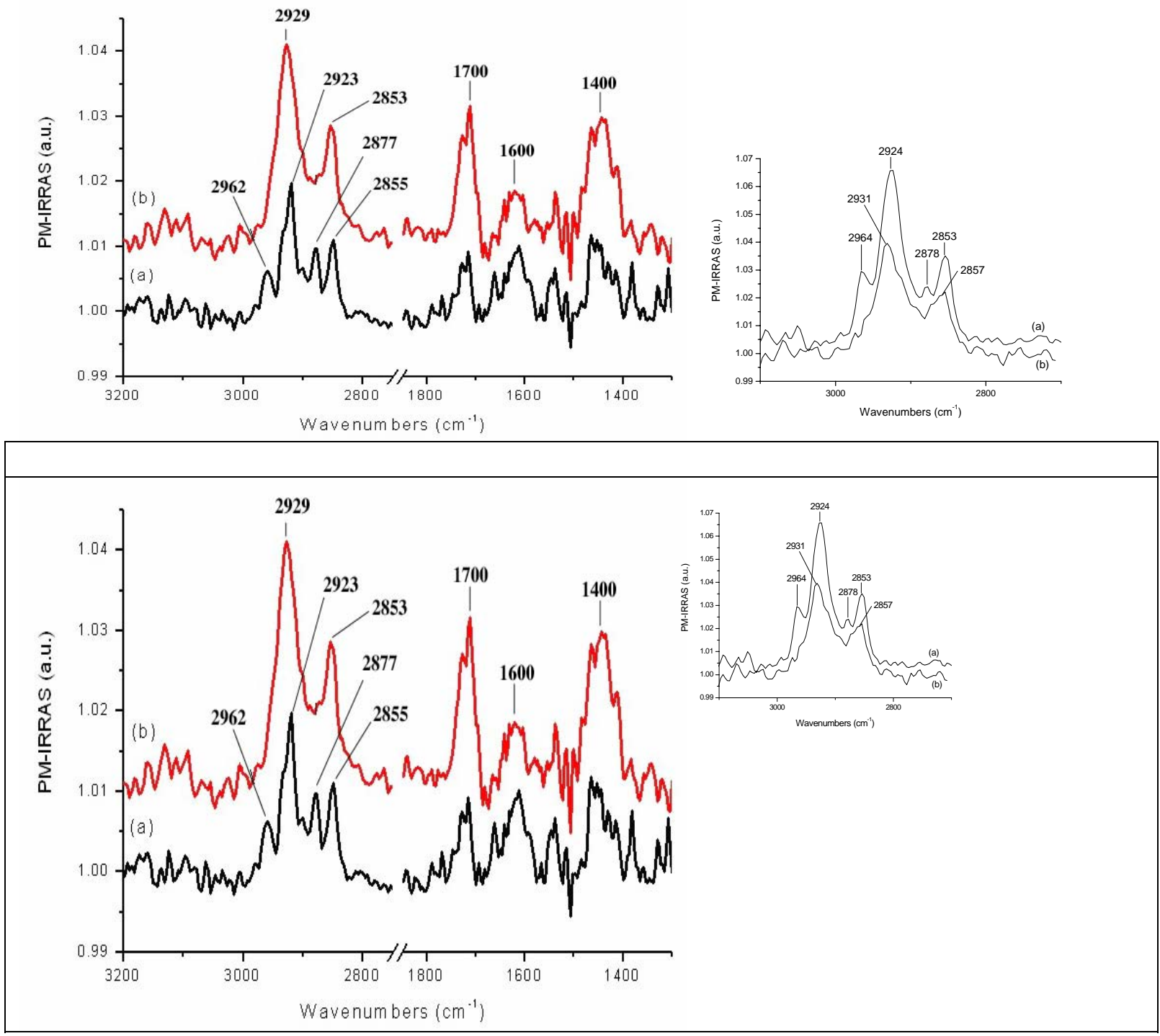
Figure 3: Topographic and phase images (insets) of the gold surfaces after immersion in mixtures of (A) MUA and C9CH3; (B) MUA and $\mathrm{C6OH}$. Samples were rinsed in ethanol and dried before analysis. Corresponding topographic cross-sections are presented below each image.

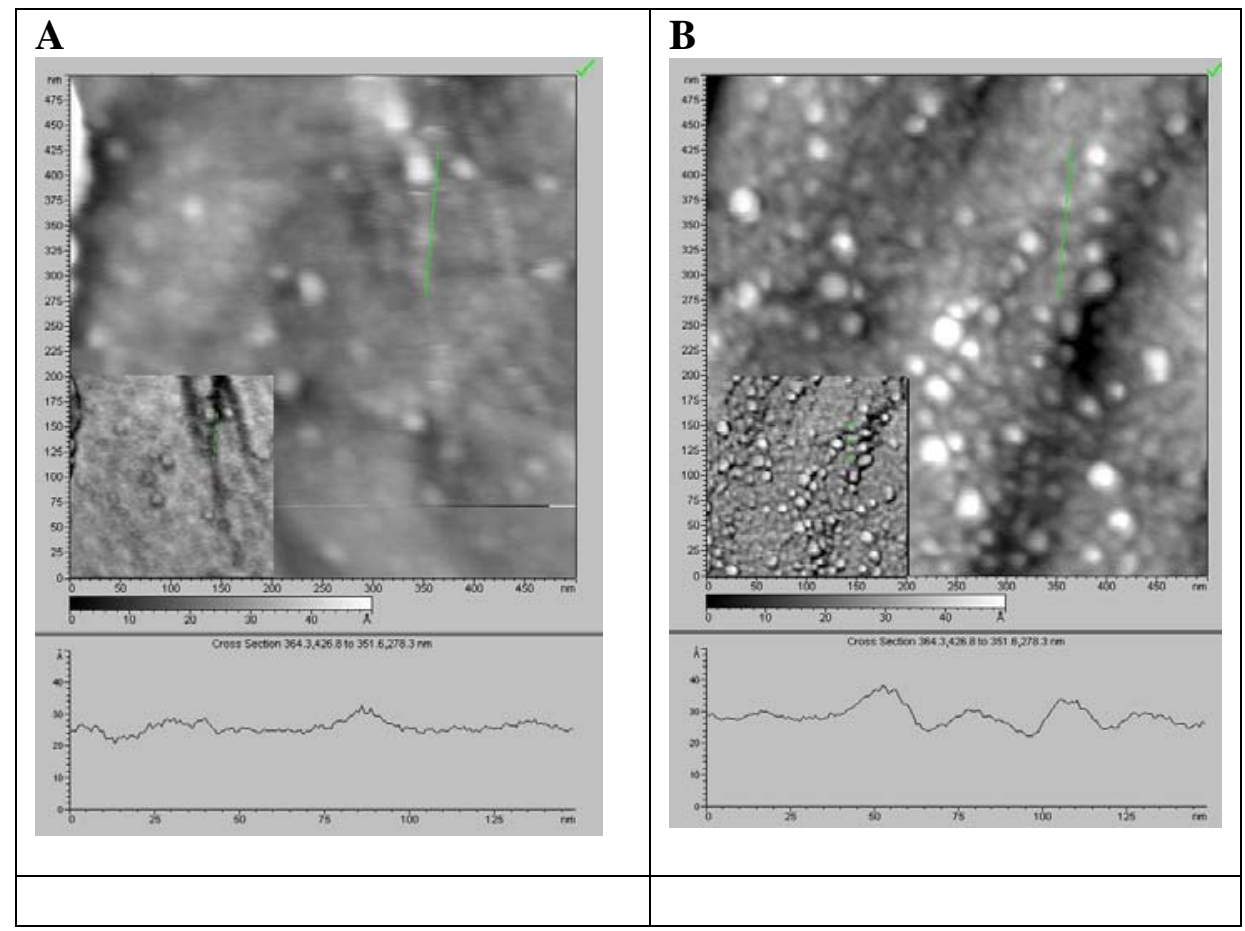

Figure 4: FT-IRRAS spectra (amide band region) of the gold surfaces after exposure to Protein A ( $0.5 \mathrm{mg} / \mathrm{L}$ in PBS, $15 \mathrm{~min})$ of the mixed SAM of (a) MUA and C9H3; (b) MUA and $\mathrm{C6OH}$. Samples were rinsed in pure water and dried before analysis.

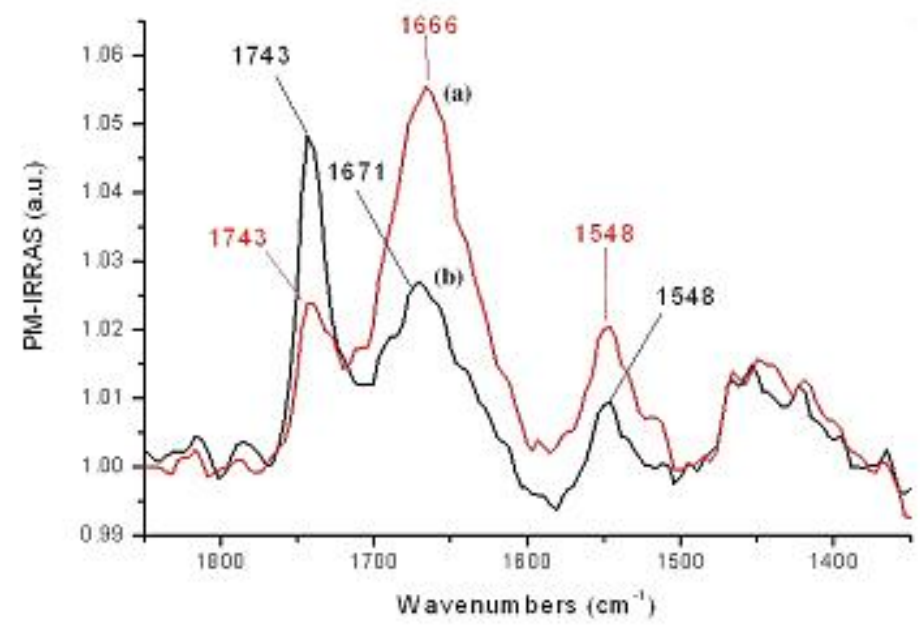


Figure 5: Topographic and phase images (inset) of functionalized gold surfaces after adsorption of Protein A on the mixed SAM of (A) MUA and C9CH3; (B) MUA and C6OH. Samples were rinsed with pure water and dried before analysis. Corresponding topographic cross-sections are presented below each image.

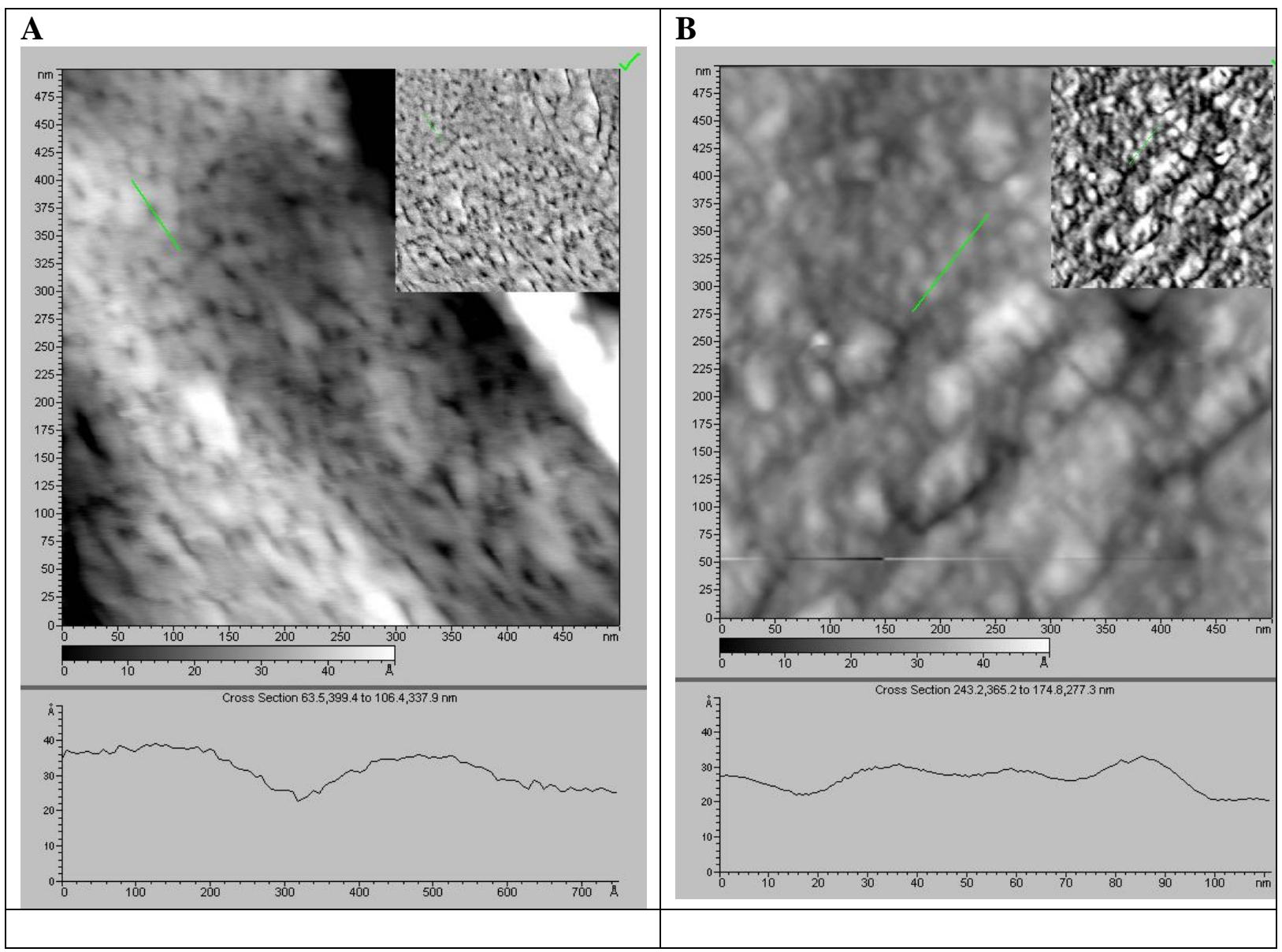

Figure 6: FT-IRRAS spectra (amide band region) of the gold surfaces after (a) Protein A adsorption; (b) blocking by BSA; (c) rabbit IgG binding. (A) Mixed SAM of MUA and C9CH3. (B) Mixed SAM of MUA and C6OH.
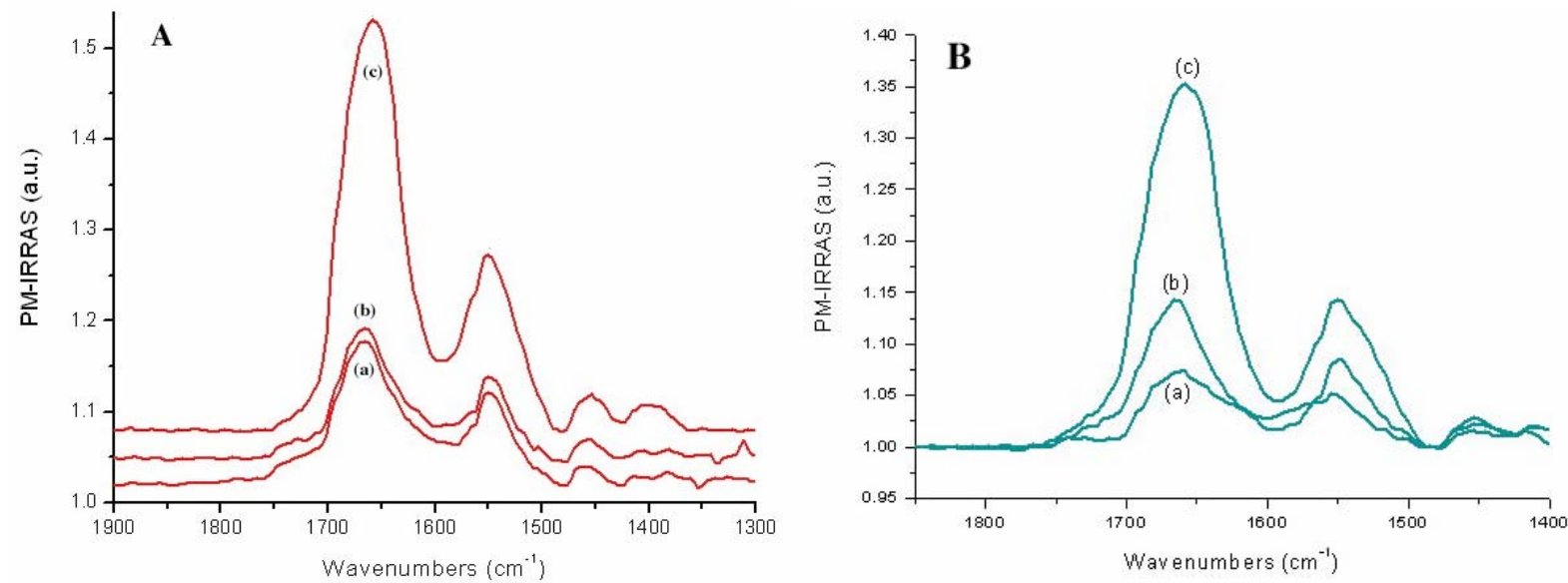
Figure 7: Frequency changes upon treatment of the functionalized gold surfaces in IgG solution, measured by in situ QCM.
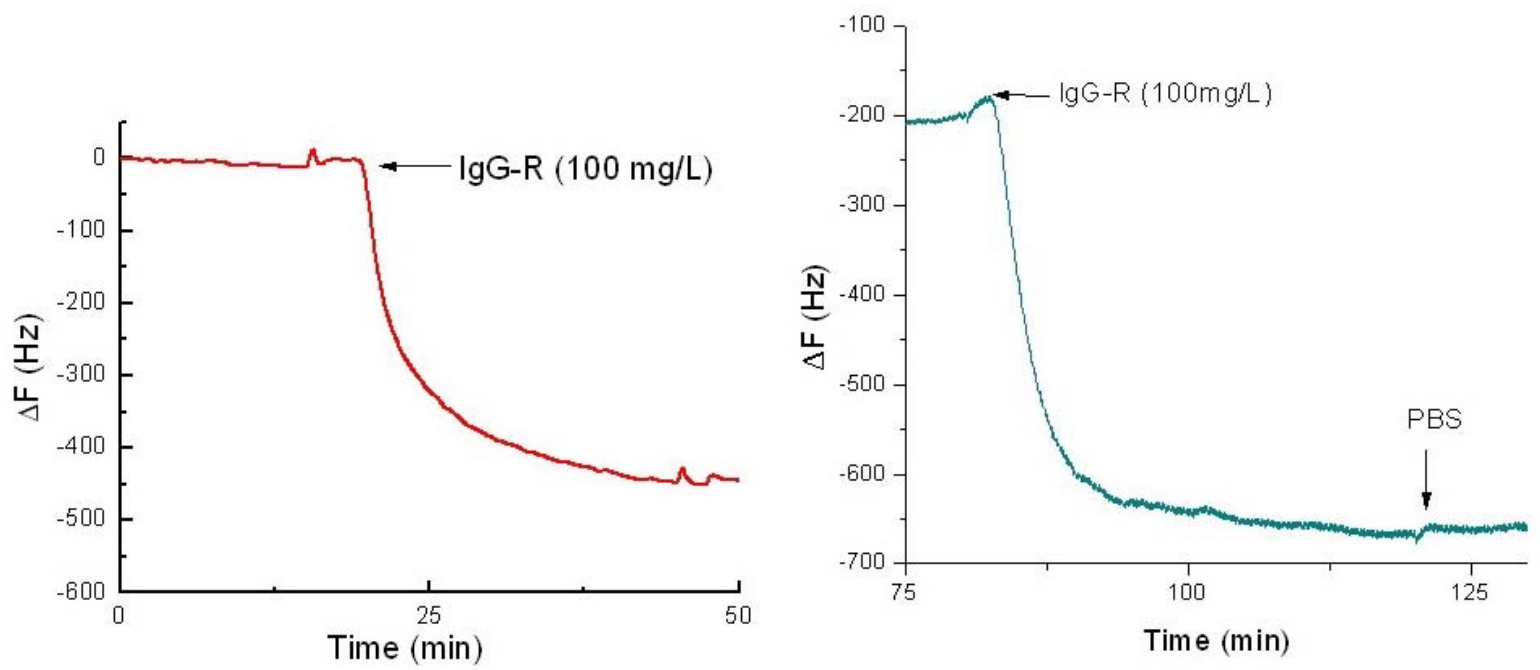

Figure 8: FT-IRRAS spectra (amide band region) of the gold surfaces after (a) Protein A adsorption; (b) rabbit IgG binding; (c) exposure to non specific anti-IgG; (d) exposure to specific anti-IgG. Mixed SAM of MUA and C6OH. Samples were rinsed with water and dried before analysis.
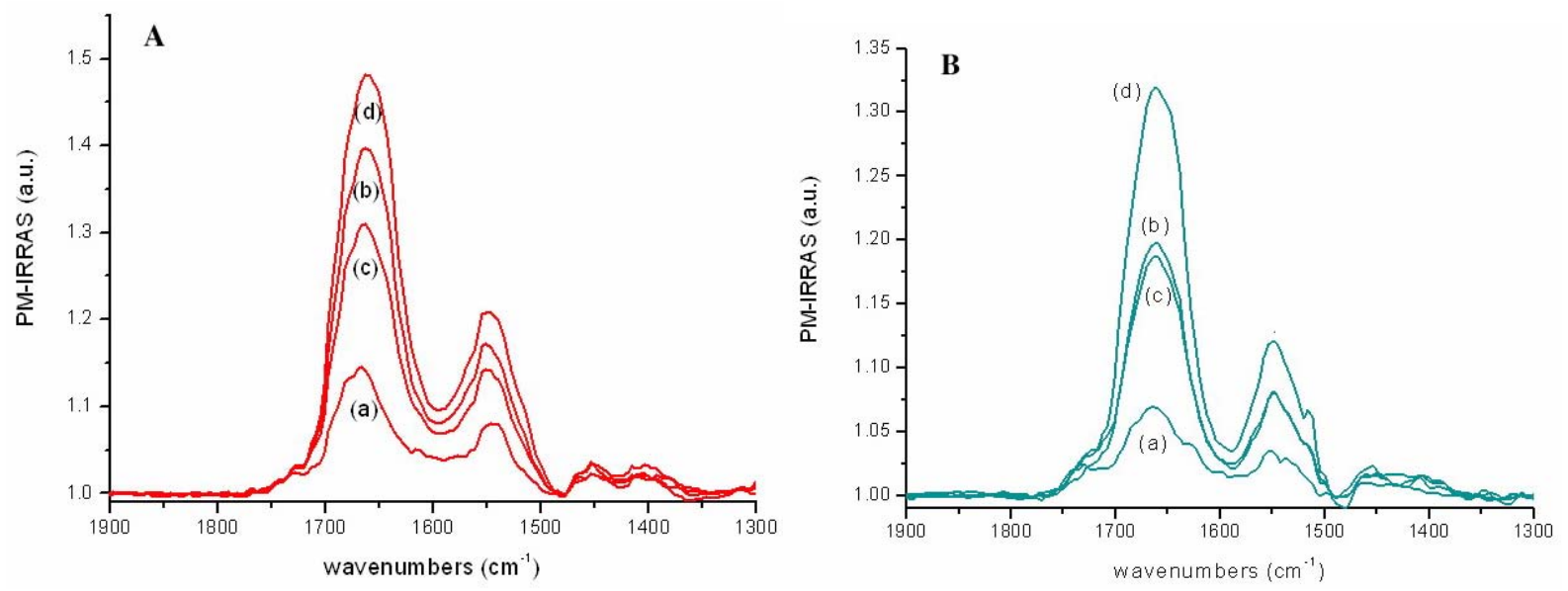